\title{
VIRUS-LIKE-PARTICLES FOR NEXT GENERATION MICRO/NANO-BIOSENSORS
}

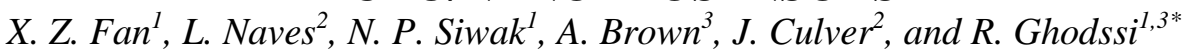 \\ ${ }^{1}$ MEMS Sensors and Actuators Laboratory, Departments of Electrical and Computer Engineering, \\ Institute for Systems Research, ${ }^{2}$ Institute for Bioscience and Biotechnology Research, \\ ${ }^{3}$ Fischell Department of Bioengineering, \\ University of Maryland, College Park, MD 20742, USA
}

\begin{abstract}
A novel Virus-like-particle (VLP) bioreceptor layer has been integrated with an optical microdisk resonator platform for biosensing applications. This bioreceptor layer can be genetically programmed for selective binding. An optical microdisk resonator has been functionalized for antibody binding with this receptor, demonstrating resonant frequency shifts of $\left(\Delta f_{o}\right)$ of $0.79 \mathrm{~nm}$ and $5.95 \mathrm{~nm}$ after primary antibody binding and full enzyme linked immunosorbent assay (ELISA), respectively. These shifts are more than $300 \%$ higher compared to the non-specific binding of a control (non-conjugated) bioreceptor layer. The sensitivity of the optical resonator to detect primary antibody binding without the additional ELISA steps demonstrates the feasibility of this platform for rapid and simplified immunoassays on a chip.
\end{abstract}

\section{INTRODUCTION}

The detection and identification of biomolecules (e.g. antibodies, proteins, and peptides) are important for a wide range of biological sciences and bio-medical applications. The primary goals and challenges shared by all sensor systems are to achieve high sensitivity and selectivity. Recently, considerable research efforts have been focused toward developing miniaturized sensor microsystems by taking advantage of their small footprint, low power consumption, and easy read-out and integration schemes. While these microsystems also greatly benefit from the high sensitivity and reliability of microtransducers (e.g. bulk and surface acoustic waves, crystal microbalances, MEMS and optical resonators), they often lack the selectivity - the ability to recognize a specific target - because of challenges associated with expressing a functionalized receptor layer.

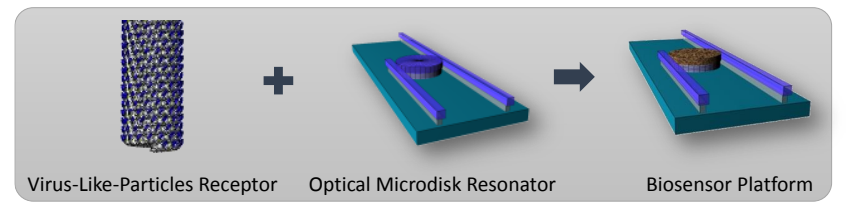

Figure 1. Schematic of the integration of VLP bioreceptor layer with an optical microdisk resonator to realize a biosensor system

The enabling technology in this work is a highly robust and genetically engineered bio-nanostructure called Virus-like-particle (VLP). VLPs are high-surface area biomolecules featuring a wide variety of genetically programmable functional groups [1-2]. These include cysteines that facilitate self-assembly onto various substrates and peptides with high affinity to target molecules. The expression of these highly selective VLP bioreceptors on the active surface of sensitive refractive index (RI) optical microresonators results in an ideal versatile platform for on-chip biosensing (Figure 1). The realization of this biosensor system was used to investigate and perform enzyme-linked immunosorbent assay (ELISA) for the detection of Flag antibody, using an established model antigenantibody system. The ultra-high sensitivity of this sensor platform has the potential to recognize this immunoassay in a label-free manner, simplifying traditional ELISA procedures.

\section{PRINCIPLE OF OPERATION}

Figure 2 shows the overview schematic of the sensor system. An optical microdisk resonator is functionalized with the VLP layer to conduct antibody sensing via ELISA. The microdisk transducer is based on a change in the refractive index of the receptor layer upon the attachment of analytes on the surface of its optical resonant cavity. These types of sensors provide high sensitivity, and real-time measurement without labeling (e.g. fluorescent, enzyme indicator), requisites in both aqueous and dry conditions. A change in the surface condition of the resonator (cladding index) due to analyte binding onto this receptor layer will cause a change in effective RI $\left(n_{\text {eff }}\right)$ of the sensor, inducing a measurable shift in resonant frequency $\left(\Delta f_{o}\right)$. As a result, by acquiring the optical spectrum of the optical sensor and tracing its $\Delta f_{o}$, surfacing binding events can be monitored.

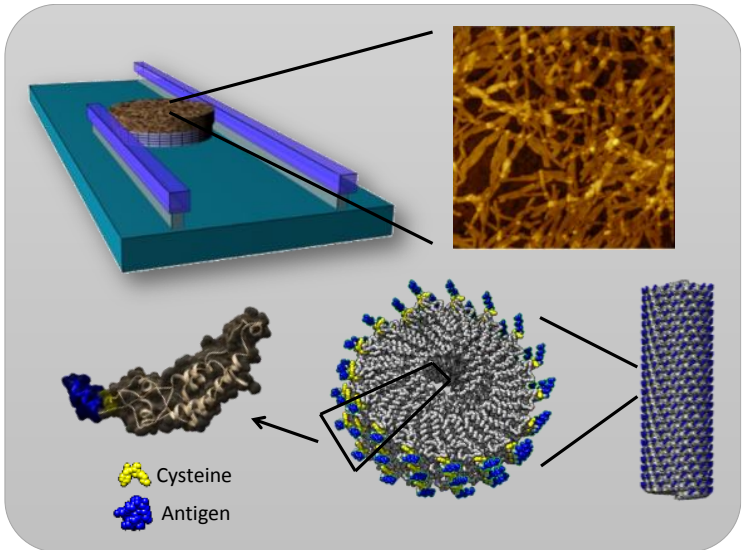

Figure 2. Schematic showing the expression of VLP on a microdisk resonator. The VLP nanostructure consists of identical CP subunits assembled into a helical formation. Each CP can be conjugated with a multitude of motifs, including cysteines (shown in yellow) and antigen peptides (shown in blue) used in this work.

We have developed the VLP bioreceptor layer for microscale selective sensing. Its selectivity may be tailored via genetic engineering. The VLP has a high aspect ratio nanotube structure formed from coat protein (CP) subunits. The VLP constructs were created in E. Coli DH5 alpha cells and expressed in BL21 bacterial cells. Each CP was genetically mutated to allow for the selfassembly of viruses onto surfaces via a cysteine ( $n C y s$ ) conjugation. An additional VLP construct with a second peptide (cFlag) sequence (DYKDDDDK) is expressed for the binding of a specific antibody to the CP's outer surface.

The coating of the microdisk transducer surface with VLP$n C y s-c F l a g$ nanotubes via cysteine binding allows for a high surface area bioreceptor layer that expresses high affinity towards Flag antibodies. This model antigen-antibody binding system is investigated by performing ELISA on this sensor chip and monitoring the optical $\Delta f_{o}$ induced by the attachment of the antibodies and the presence enzymatic activities. 


\section{DESIGN AND METHODS}

Figure 3 (a) shows the abridged fabrication process flow of the microdisk resonator. The $20 \mu \mathrm{m}$ diameter and $340 \mathrm{~nm}$ thick silicon nitride on oxide microdisks were patterned using E-beam lithography. The $300 \mathrm{~nm}$ coupling gap and $600 \mathrm{~nm}$ wide waveguides extended to the edges of the chip and were optimized for system sensitivity. Reactive ion etching was used to transfer the resist pattern into an oxide mask and subsequently into the waveguide layer and was optimized for vertical and smooth sidewalls. The 3 $\mathrm{mm}$ wide chips were cleaved to ensure crystalline facets and minimal optical losses when light is coupled on to and off of the chip from lensed fibers. The fabricated microdisks were characterized using an IR tunable laser (1520-1620 nm).

Two types of VLPs were assembled on different microdisk resonator chips. VLP- $n C y s-c F l a g$ was used to selectively bind to antibodies, and VLP- $n C y s$ (without the selective peptide) was used as a negative control to monitor unspecific bindings. Both chips underwent identical ELISA procedures by submerging the chip in solution, allowing for a sequential enzymatic binding of primary antibody (anti-Flag), secondary antibody, and enzymatic indicator assembly (schematically shown in Figure 3 (b)). The optical spectrum of each sensor chip was acquired under identical dehydrated conditions and the resonant frequency of the pre and post-ELISA of the two transducers were compared. Label-free immunoassays were conducted by introducing only primary antibody without secondary antibody and enzymatic indicator labeling. The selectivity of the bioreceptor layer was investigated against non-complementary antibodies (anti-HA and anti-His) as well as $5 \%$ non-fat milk solution, which contains a host of proteins and salts that can contribute to non-specific binding.

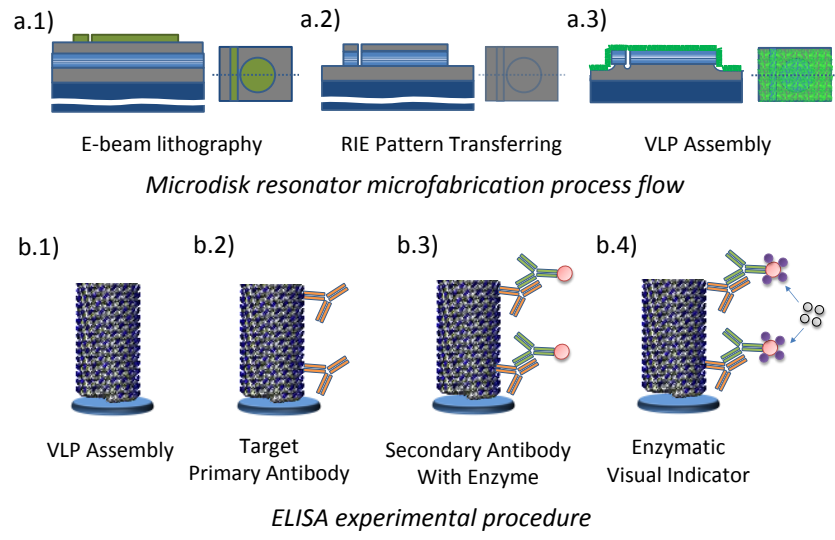

Figure 3. (a.1-3) An abridged schematic of the microdisk resonator fabrication process flow and (b.1-4) sequential steps of the ELISA experimental procedure conducted

\section{RESULTS}

The sensitivity of the transducer based on the stability and noise level of the device and setup over a period of 24 hours was $2.11 \mathrm{pm}$. As seen from Figure 4, the assembly of the VLP-nCyscFlag and VLP- $n C y s$ on microdisk resonators induced a $\Delta f_{o}$ of $1.05 \pm 0.23 \mathrm{~nm}$ and $2.21 \pm 0.34 \mathrm{~nm}$, respectively. The difference in wavelength shift is attributed to the difference in coverage density between the two strands of VLP, which was also confirmed by SEM (not shown here). This is most likely due to steric hindrance effects in the VLP-nCys-cFlag due to the additional peptide conjugated onto its surface. The full ELISA procedure shifted the resonant frequency by $+5.95 \mathrm{~nm}(567 \%$, with respect to receptor layer assembly) and $-0.08 \mathrm{~nm}(-4 \%)$ on VLP-nCys-cFlag and VLP- $n$ Cys coated sensor chips, respectively. The difference in the $\Delta f_{o}$ indicates the selective bonding of Flag antibody to the genetically conjugated cFlag binding sites. Simplifying and truncating the ELISA process, label free detection of only the Flag antibody showed a $\triangle f_{o}$ of +0.79 $\mathrm{nm}(51 \%)$. The selectivity of $V L P-n C y s-c$ Flag coated sensor chips were verified against non-complementary antibody HA and antibody His and both resulted in a negative shift in resonant frequency. The exposure to $5 \%$ non-fat milk also showed no unspecific binding to the receptor layer, showing a $\Delta f_{o}$ of $-0.03 \mathrm{~nm}$ $(-7.1 \%)$. The sensor was, however, able to detect the positive presence of the antibody, $\Delta f_{o}=+0.13 \mathrm{~nm}(34.8 \%)$, when the milk solution was mixed with Flag antibody at 1:1000 dilution. The negative shifts in resonant frequency are all associated with the rinsing and drying steps of the experimental procedure and were verified in an independent experiment.

The results presented here demonstrate the capability of a VLPbased receptor layer whose genetically programmable coat protein can display a multitude of unique binding motifs on transducer surfaces for target analytes. The sensitivity of the platform enabled, for the first time, utilization of VLP for label-free detection and simplification of the standard ELISA process and conduct, decreasing time and cost of immunoassays and creating new sensing opportunities.

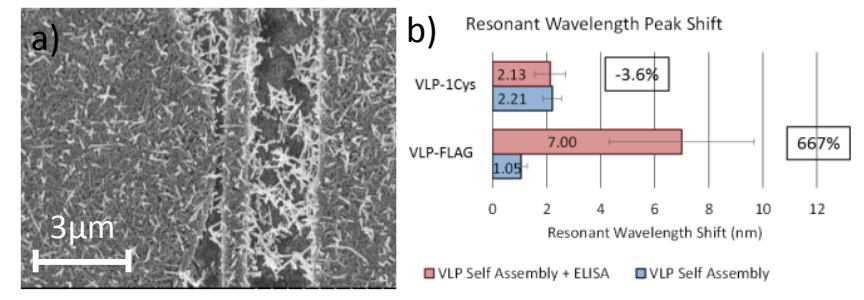

Figure 4. (a) A SEM image showing the assembly of VLPs on the surface of a micirodisk resonator (VLPs are metal coated for imaging). (b) Resonant frequency shift of pre- and post-ELISA on VLP-n1Cys and VLP-nlCys-cFlag coated sensor chips.

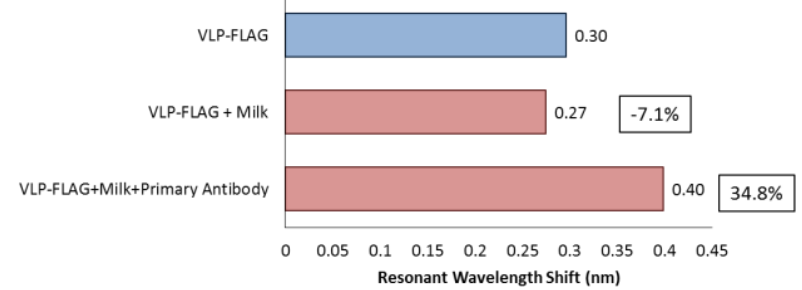

Figure 5. Resonant frequency shift of sensor chip after submersion in milk, $-7.1 \%$, and milk with primary Flag antibody, $+34.8 \%$, demonstrating the high selectivity of VLP-n1Cys-cFlag.

\section{REFERENCES}

[1] A. Brown, L. Naves, X. Wang, R. Ghodssi, and J. N. Culver, "Carboxylate Directed In Vivo Assembly of Virus-Like Nanorods and Tubes for the Display of Functional Peptides and Residues," Biomacromolecules, vol. 14, pp. 3123-3129, July 2013.

[2] X. Z. Fan, E. Pomerantseva, M. Gnerlich, A. Brown, K. Gerasopoulos, M. McCarthy, J. Culver, and R. Ghodssi, "Tobacco mosaic virus: a biological building block for micro/nano systems," Journal of Vacuum Science and Technology A, vol. 31, pp. 050815-1-24, August 2013 Invited

\section{CONTACT}

*Professor Reza Ghodssi: Tel.: +1 301405 8158; E-mail: ghodssi@umd.edu 